\title{
An outline of the programme of rehabilitation medical services in the People's Republic of China: 1991-1995
}

\author{
Chen Zhong-Wu MD \\ Ministry of Public Health, People's Republic of China, 44 Houhai Beiyan, Beijing, \\ China.
}

An Outline of the Programme of Rehabilitative Medical Services, 1991-1995 was jointly promulgated on 31 July 1991 by the Ministry of Public Health, the Ministry of Civil Affairs, and the Chinese Association for the Handicapped of this country.

Basing itself on the analysis of the present status, the Outline has defined: (1) the basic tasks and specific goals of this country for the development of rehabilitative medical services covering the period 1991-1995; and (2) its policies and principal measures.

The Outline specifies that prominence is to be given to the training of professionals in rehabilitation medicine, and that training is to be organised in all forms in order to provide favourable conditions for the gradual formation in this country of a rehabilitation medical service. Training centres for rehabilitation medical professionals are to be organised in provinces, municipalities and autonomous regions where there are suitable conditions. Rehabilitation medicine is to be taught as a subject in medical colleges, secondary medical schools and nurse training schools; whereas faculties in rehabilitation medicine and rehabilitation therapy are to be set up in institutions wherever possible, and are to be associated with the working plans of departments of education, of public health, and of civil affairs. At the same time, the in-service training is to be strengthened to further raise the expertise of rehabilitation medical professional staff. It is imperative to bring into play the initiatives of the existing rural and urban medical networks in the development of rehabilitation medical institutions. Of the general hospitals affiliated to the provincial municipal autonomous regional department and of the teaching hospitals of medical colleges, one quarter are expected to initiate pilot studies in rehabilitation medical services. In the general hospitals where conditions are more favourable, units of rehabilitation medicine are expected to be set up as technological guiding centres. The Outline also requires the expansion of coverage of community rehabilitation medical services to benefit a large number of rural and urban handicapped persons. This would be promoted at street committee level in large sized municipalities, at district/ street committee level in medium and small sized cities, and at township level in the rural areas. 